\title{
Plasma Heating by Antiproton Annihilation in Magnetic Mirror Fusion Propulsion
}

\author{
B. Cassenti* \\ United Technologies Research Center \\ East Hartford, CT \\ Terry Kammash** \\ University of Michigan \\ Ann Arbor, MI
}

\begin{abstract}
Magnetic mirror fusion propulsion shows promise as a means for opening up interplanetary travel. The systems, though, tend to be large and heavy. One of the heaviest components in the system is the plasma heater. Heating by the annihilation of antiprotons in the plasma could result in a considerable reduction of mass. An examination of various heating sources in the annihilation reaction show that most of the heating may not be effective. Although, heating by the relativistic electrons may be useful the plasma chamber radius and lengths may have to be increased. An indirect heating method, where antiprotons are annihilated in a fissionable material, such as uranium- 238 , may be useful. The resulting fission products are then used to heat the plasma, which should be more efficient.

\section{Introduction}

Magnetic mirror fusion[1] was the subject of intense research in the early 1960's. Although mirror machines showed a remarkable resistance to macroscopic plasma instabilities they were prone to develop microscopic instabilities. In the 1970's it was noted that the stability of magnetic mirror devices could be increased by increasing the plasma density, while improved designs for the

* Senior Principal Engineer, Associate Fellow AIAA

* Professor, Associate Fellow AIAA
\end{abstract}

magnetic fields were also incorporated.

Unfortunately, mirror devices could not compete with tokamaks and are not now seriously considered as commercially viable devices.

Early magnetic mirror fusion devices not only suffered from microscopic instabilities, but also can leak plasma at a substantial rate. This increases the heating requirements and hence decreases the overall efficiency as a power generator, but this leakage is exactly what is required for a deep space propulsion system. The higher plasma densities, to combat micro instabilities, will increase the thrust, enhancing the performance as a propulsion system. Kammash and Lee[2] and Emmrich[3] have shown that a propulsion system based on magnetic mirror fusion would provide a substantial increase in performance. The device, see Fig. 1, traps the ions radially using the central coils, and also forms a longitudinal trap by applying higher magnetic intensities at the ends.

In magnetic mirror fusion devices plasma particles that are moving nearly parallel to the axis of the device will leak out the ends. The particles leaking out one end can be used for the exhaust, as in a conventional chemical rocket. The particles leaking out the other end can be used for energy. This energy can be used to partially heat the plasma in the mirror device. The total heat to be added, to create the plasma in the mirror, is substantial, and could amount to one-third of heat generated in the fusion reaction. The equipment required to heat the plasma will be massive[2,3] and should approach one-quarter of the total vehicle mass.

Copyright (C)1997 by the American Institute of Aeronautics and Astronautics, Inc. All rights reserved. 
One method of heating the plasma that would require significantly less mass would be to store the energy in the form of antiprotons. The annihilation energy then could be used to heat the plasma. The mass required to store and inject the antiprotons could be substantially less, and the depth at which the antiprotons annihilate can be accurately controlled[4] to insure the annihilation heating will occur at the center of the chamber.

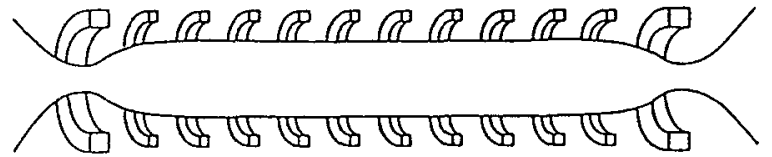

Fig. 1 Magnetic Mirror Fusion System

Antiproton heating is not a new idea. Cassenti[5] and Howe and Metzger[6] in the mid 1980's proposed using antiprotons to heat a gaseous propellant. These systems though require 10's of milligrams of antiprotons for a typical mission. Antiprotons annihilate into pions (with about 5 percent kaons.) The pions have a mean life of 26 nsec and decay into muons and neutrinos. The muons decay with a mean life of $2.2 \mathrm{msec}$ and decay into electrons (or positrons) and neutrinos. The short life of the pions and muons may not allow sufficient heating before the decay, but the electrons and positrons created carry 18 percent of the original annihilation energy[5], if the annihilation occurs in a vacuum. The high energy electrons created in the annihilations have an energy distribution that can be readily determined and the losses for the electrons can be also determined. There were three energy losses that were examined: 1) ionization, i.e., the heating of the plasma, 2) bremsstrahlung and 3) synchrotron losses. The bremsstrahlung and synchrotron losses contribute little energy to the plasma.

In addition to heating from the annihilation products, some of the pions will be immediately absorbed in a nucleus consisting of more than one nucleon. In our case, these are deuterium and tritium. Finally, if very heavy nuclei are present, such as uranium or plutonium, they will fission[7]) producing high energy, high mass, fission nuclei that will readily deposit their energy in the plasma.

The above heating mechanisms will each be discussed in detail below.

\section{Pion/Muon Heating}

In an annihilation of an antiproton and a proton, or a neutron, the pions are released at about 400 $\mathrm{MeV}$ total energy. At $400 \mathrm{MeV}$ the pions lose energy in liquid hydrogen at a rate of 0.15 $\mathrm{MeV} / \mathrm{cm}[8]$. Liquid hydrogen has a density of $4.2 \times 10^{24} / \mathrm{cm}^{3}$, but densities in a gasdynamic mirror propulsion system will be about $1 \times 10^{15}$ to $1 \times 10^{18} / \mathrm{cm}^{3}$. This means the loss would be, at most, about $4 \times 10^{-8} \mathrm{MeV} / \mathrm{cm}$. The pion would go about 20 meters before decaying (including the relativistic increase in the mean life) and would deposit, at most, about $1 \times 10^{-4} \mathrm{MeV}$, a truly negligible amount.

The muons resulting from the pion decay would have an energy of about $300 \mathrm{MeV}$ [9], and again would lose energy in liquid hydrogen at a rate of $0.15 \mathrm{MeV} / \mathrm{cm}[8]$. The muons would go about two kilometers before decaying. Hence, the muons would deposit in the plasma about $0.05 \mathrm{MeV}$, and again this is negligible.

Hence, we can safely conclude that the pions and muons should contribute little heating to the plasma.

\section{Electron Heating}

The decay of the muons will result in the emission of energetic electrons and positrons. If the antiproton annihilation occurred in a vacuum, the electrons and positrons would carry about 18 percent of the annihilation energy [5]. The remaining energy would be lost to the neutrinos produced in the decay of the muons. The Monte-Carlo simulations described in References[5] and $[9]$ were repeated and the result for 1000 pion decays in a vacuum are shown in Figure 2. The average energy of the electrons (throughout the remainder of this paper electrons will refer to both electorns and positrons) was $105 \mathrm{MeV}$ and is well described by

$$
p(E)=\frac{a}{\Gamma(n+1)}(a E)^{n} e^{(-a E)}
$$

where $E$ is the energy of the electron, $p(E) d E$ is the energy between $E$ and $E+d E$, and $a$ and $n$ 
are parameters. The parameters $a$ and $n$ can be readily shown to be related to the average energy, Eavg, by

$$
E_{\text {avg }}=\frac{n+1}{a}
$$

The function $\Gamma$ in equation (1) is the gamma function, were

$$
\Gamma(n+1)=n !
$$

For the distribution in Figure 1 the average energy is $105 \mathrm{MeV}$ and the parameter $\mathrm{n}$ is 0.5 . From Tollman[10] the temperature can be found from

$$
\frac{k T}{m c^{2}}=\frac{1}{3}\left(\frac{E_{a v g}}{m_{e} c^{2}}-\frac{m_{e} c^{2}}{E_{a v g}}\right)
$$

where $m_{e} c^{2}$ is rest mass energy of the electron $(0.511 \mathrm{MeV})$, and $K T$ is the temperature. For an average energy of $105 \mathrm{MeV}$, the corresponding temperature is $35 \mathrm{MeV}$. It should be noted that the fit in equation (1) is not the correct form for a highly relativistic gas $[10,11]$.

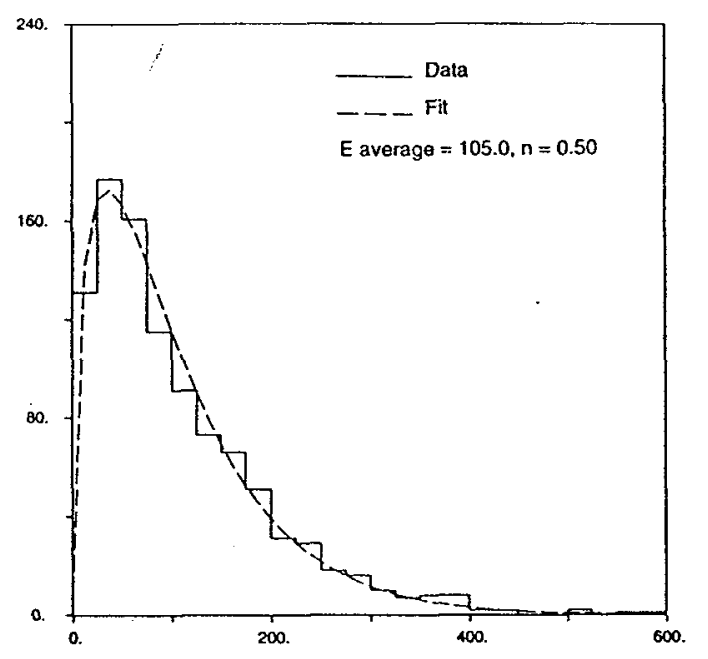

Fig. 2 Electron Energy Distribution

The relativistic electrons will lose energy as it moves through the plasma by: 1) ionization, 2) Bremsstrahlung radiation and 3 ) by Synchrotron loses. Bremsstrahlung loses occur due to the acceleration of an electron as it passes the ions in the plasma and synchrotron loses occur when the electrons accelerate in a magnetic field.

The electron ionization loses can be accurately found from[12]

$$
\begin{gathered}
\frac{d E_{i}}{d t} \approx \frac{2 \pi n_{i} e^{4}}{m_{e} c \sqrt{\gamma+1}}\left(\frac{\gamma^{2}}{R}\right) \sqrt{\frac{m_{e} c^{2}}{k T_{p l}}} \\
\ln \left[\frac{m_{e} c^{2}}{k T_{p l}}\left(\frac{\hbar c}{m_{e} c^{2} \lambda_{D}}\right)^{2}\right]
\end{gathered}
$$

where $n_{i}$ is the ion density, $e$ is the charge on the electron, $\hbar$ is Planck's constant divided by $2 \pi, \gamma$ is the electron energy divided by its rest mass energy, $c$ is the speed of light in a vacuum, $k T_{p l}$ is the plasma temperature, $\lambda_{D}$ is the Debye shielding length, and $R$ is a dimensionaless parameter, given by

$$
R=\sqrt{\frac{m_{e} c^{2}}{k T_{p l}}\left(\gamma^{2}-1\right)}
$$

while the Debye shielding parameter for, deuterium tritium plasmas, can be found from

$$
\lambda_{D}=\sqrt{\frac{k T_{p l}}{n_{i} c^{2}}}
$$

Substituting for $R$, and $\lambda_{D}$ in equation (4)

$$
\begin{gathered}
\frac{E_{i}}{d t} \approx \frac{2 \pi n_{i} e \gamma^{2}}{m_{e} c \sqrt{\gamma^{2}-1}} \\
\ln \left[\left(\frac{m_{e} c^{2}}{k T_{p l}}\right) \frac{n_{i} e^{2}(\hbar c)^{2}}{\left(m_{e} c^{2}\right)^{3}}\right]
\end{gathered}
$$

For highly relativistic particles, $\gamma^{2}>>1$, and the energy loss is proportional to the energy.

The Bremsstrahlung losses can be estimated by assuming that the electron travels in a straight line as it passes an ion. Since the Bremsstrahlung loss is given by[12].

$$
\frac{d e_{b}}{d t}=\frac{2}{3} \frac{(e Z) a^{2}}{c^{3}}
$$

where $Z$ is the charge on the ions (for hydrogen $Z$ is one) and $a$ is the acceleration of electron, which is given by

$$
a=\frac{e^{2}}{m_{e} r^{2}}
$$

Taking the distance, $\mathrm{r}$, to be a constant, equations (9) and (10) yield 


$$
\frac{d E_{b}}{d t} \approx \frac{2 e^{6}}{3 m_{e}^{2} c^{3} r^{4}}
$$

Let the probability for $r$ to be between $r$ and $r+d r$ be $p(r) d r$ and take $r$ to vary from $r_{\min }$ to $r_{0}$, where $r_{0}$ is half the average distance between ions, or

$$
r_{0}=\frac{1}{2}\left(\frac{1}{n_{i}}\right)^{1 / 3}
$$

while $r_{\min }$ will be taken to be the electron Compton wavelength, or

$$
r_{\min }=\frac{\hbar}{m_{e} c}
$$

Assume the probability distribution is uniform and given by

$$
p(r) d r=\frac{2 \pi r d r}{\pi r_{0}^{2}}
$$

Integrating over $r$ from $r_{\min }$ to $r_{0}$

$$
\frac{d E_{b}}{d t}=\frac{8 e^{6} c n_{i}^{2 / 3}}{3(\hbar c)^{2}}
$$

The electron synchrotron radiation losses in the magnetic field of the magnetic mirror fusion device can be estimated by using[12]

$$
\frac{d E_{s}}{d t}=\frac{2}{3} \frac{e^{2} v^{4}}{c^{3} R^{2}}
$$

where $v$ is the speed of the electron (essentially the speed of light, $c$ ) and $R$ is the radius of the electron's motion in the magnetic field of field strength, $B$. For highly relativistic electrons

$$
R^{2}=\frac{\gamma^{2} m_{e}^{2} c^{4}}{e^{2} B^{2}}
$$

Then equation (14) becomes

$$
\frac{d E_{s}}{d t}=\frac{2 e^{4} B^{2} c}{3\left(m_{e} c^{2}\right)^{2}}
$$

Summing equations (7), (13), and (16) the total energy/loss is

$$
\frac{d E}{d t}=m_{e} c^{2} \frac{d \gamma}{d t}=\left(f_{b}+f_{i} \gamma+f_{s} \gamma^{2}\right) m_{e} c^{2}
$$

where

$$
\begin{gathered}
f_{i}=\frac{2 \pi n_{i} e^{4} c}{m_{e}^{2} c^{4}} \ln \left[\frac{\hbar c}{k T_{p l}} \sqrt{\frac{n_{i} e^{2}}{m_{e} c^{2}}}\right] \\
f_{b}=\frac{8 e^{6} n_{i}^{2 / 3} c}{3(\hbar c)^{2} m_{e} c^{2}} \\
f_{s}=\frac{2 e^{4} B^{2} c}{3 m_{e}^{3} c^{6}}
\end{gathered}
$$

The distance traveled before stopping can be found using

$$
\frac{d \gamma}{d t}=\frac{1}{c} \frac{d \gamma}{d s}
$$

Integrating equation (17) using equation (21) the length traveled by the electron is given by

$$
L=c \int_{1}^{\gamma_{0}} \frac{d \gamma}{f_{s} \gamma^{2}+f_{i} \gamma+f_{b}}
$$

where

$$
\gamma_{0}=\frac{E_{0}}{m_{e} c^{2}}
$$

and $E_{0}$ is the initial energy of the electron.

Although equation (22) can be readily integrated, an examination of the terms in equation (23) for a gasdynamic mirror propulsion system[2,3] will demonstrate that

$$
f_{s} \gamma_{0}^{2}<<f_{i} \gamma_{0}+f_{b}
$$

Hence, the synchrotron radiation losses can be neglected. In fact, an examination of the losses over a wide range of energies indicates that the ionization losses dominate over most of the applicable energies, and conditions.

An estimate for the length required for the energy to be deposited can be readily obtained by only including the ionization losses then equation (22) yields

$$
L=\frac{c}{f_{i}} \ln \gamma_{0}
$$

Taking

$$
\gamma_{0}=\frac{E_{a v g}}{m_{e} c^{2}}=205
$$

Table I illustrates the deposition lengths, $L$, for various mirror parameters including ionization and Bremsstrahlung loses. 
Table 1 Electron Energy Deposition Lengths

$\begin{array}{ccc}\text { Temperature } & \text { Density } & \text { Lengths } \\ \text { KeV } & 1 / \text { cm }^{3} & m \\ 5 & 1 \times 10^{16} & 6500 \\ 5 & 1 \times 10^{17} & 700 \\ 5 & 1 \times 10^{18} & 77 \\ 10 & 1 \times 10^{16} & 6300 \\ 10 & 1 \times 10^{17} & 670 \\ 10 & 1 \times 10^{18} & 73 \\ 15 & 1 \times 10^{16} & 6000 \\ 15 & 1 \times 10^{17} & 640 \\ 15 & 1 \times 10^{18} & 69\end{array}$

These lengths are somewhat long and scattering of the electrons could be important. Hence more accurate calculations need to be performed. These results indicate that the electrons may deposit appreciable energy to the plasma.

\section{Recoil Nucleon Heating}

When the antiproton annihilates on a nucleus of atomic weight two or greater one of the nucleons is annihilated, while the remaining nucleus may absorb some of the emitted pions and be ejected at high energy. Measurements of the energies of ejected nucleons(13) indicate that for deuterium the energy distribution is given by equation (1) with,

$$
a=\frac{1}{66} M_{e V}^{-1}
$$

and

$$
n=0
$$

for nonrelatixistic protons. The average energy of the protons is then, from equation (2)

$$
E_{\text {avg }}=66 \mathrm{MeV}
$$

In a mixture of deuterium and tritium assume one half of the annihilations will occur on the deuterium nucleus and the other half on the tritium nucleus. An annihilation on the deuterium nucleus will yield a high energy proton half the time, while tritium will always yield a high energy proton (since deuterium nucleus has a binding energy of $2.2 \mathrm{MeV}$, which is much less than the recoil energy of the proton). For tritium conservatively assume half of the absorbed annihilation energy goes to each remaining nucleon. Then the average energy in the recoil protons will be slightly over $25 \mathrm{MeV}$.
The recoil protons are moving slowly enough to remain within the plasma chamber while their kinetics energy is absorbed. Although this recoil energy is not large, it should not be neglected.

\section{Fission Fragment Heating}

If the plasma is seeded at the center of the magnetic chamber with uranium or plutonium, then when the antiprotons annihilate on these heavy nuclei, they will fission[7] producing fission fragments with a total kinetic energy of more than $140 \mathrm{MeV}$. The fission fragments, like the protons, will deposit their kinetic energy readily in the plasma. For the use of fission to be a practical method to heat the plasma, the uranium (or plutonium) must be kept in the center of the chamber free of deuterium and tritium as in a gas core nuclear rocket. This is not trivial, as the gas core rocket has shown under less severe conditions, but the payoff could be significant.

\section{Conclusions}

Magnetic mirror fusion propulsion shows promise as a future method of space propulsion. Significant mass savings would result if the plasma heaters could be removed. Antiproton heating could help achieve this goal. Direct heating by the pions and muons will not contribute to the heating, but the electrons may provide sufficient heating and requires more refined modeling. Recoil proton heating, although not large must be included in design analyses. Finally, it may be possible to heat the plasma using fission fragments released by antiproton annihilations in uranium and plutonium.

\section{References}

1. Bromberg, J. L. Fusion - Science, Politics and the Invention of a New Energy Source, The MIT Press, Cambridge, Massachusetts, 1983.

2. Kammash, T., and M.-J. Lee, High-Thrust-High-Specific Impulse Gasdynamic Fusion Propulsion System, Journal of Propulsion and Power, 13, No. 3, pp. 421-427, 1997.

3. Emrich, W. J., Jr., Practical Interplanetary Travel Using a Gasdynamic Using a Gasdynamic Mirror Fusion Propulsion System, presented at the 32nd AIAA/ASME/SAE/ASEE Joint Propulsion Conference, Lake Buena Vista, July 1-3, 1996.

4. Kammash, T. and M.-J. Lee, Antiproton 
Annihilation Dynamics in the Gasdynamic Fusion Rocket, AIP Conference Proceedings 361, pp. 1401-1405, 1996.

5. Cassenti, B. N., Antimatter Propulsion for $O T V$ Applications, Journal of Propulsion and Power, 1, pp. 143-149, 1985.

6. Howe, S. D. and J. D. Metzger, Survey of Antiproton Based Propulsion Concepts and Potential Impact on a Mars Missions, Los Alamos National Laboratory, LA-UR-87-2191, Los Alamos, NM, 1987.

7. Lewis, R. A., G. A. Smith, R. J. Kanzlecter and R. Newton, Antiproton Based Microfusion, Fusion Technology, 20, Dec. 1991, pp. 1046-1050.

8. Particle Data Group, Review of Particle Properties, Physical Review D. 45, Part II, 1 June 1992.

9. Cassenti, B. N., Design Considerations for Relativistic Antimatter Rockets, Journal of the British Interplanetary Society, 35, 1982, pp. 396-404.

10. Tollman, R. C., The Principles of Statistical Mechanics, Dover Publications, Inc., New York, 1939.

11. Synge, J. L. The Relativistic Gas, North Holland Publishing Company, Amsterdam, 1957.

12. Kammash, T., Fusion Reactor Physics Principles and Technology, Ann Arbor Science Publishers, Inc., Ann Arbor, 1975.

13. Riedlberger, J., et al, Antiproton Annihilation at Rest in Nitrogen and Deuterium Gas, Physical Review C, 40, pp. 2717-2731, 1989. 